\title{
Fatores de risco para doenças cardiovasculares em profissionais de enfermagem: estratégias de promoção da saúde
}

\author{
Risk factors for cardiovascular diseases among nursing professionals: strategies for health promotion \\ Factores de riesgo para las enfermedades cardiovasculares entre profesionales de enfermería: \\ estrategias para promoción de la salud
}

\section{Fernanda Jorge Magalhães', Larissa Bento de Araújo Mendonça', Cristiana Brasil de Almeida Rebouças", Francisca Elisângela Teixeira Lima", Ires Lopes Custódio"II, Samya Coutinho de Oliveira"I' \\ ' Universidade Federal do Ceará, Programa de Pós-Graduação em Enfermagem. Fortaleza-CE, Brasil. \\ "Universidade Federal do Ceará, Departamento de Enfermagem. Fortaleza-CE, Brasil. \\ III Secretaria de Saúde do Estado do Ceará, Hospital Dr. Carlos Alberto Studart Gomes, Fortaleza-CE,Brasil.}

Submissão: 18-09-2012 Aprovação: 05-05-2014

\section{RESUMO}

O estudo objetivou identificar os fatores de risco modificáveis e não modificáveis para doenças cardiovasculares presentes nos profissionais de enfermagem, e descrever estratégias de promoção da saúde para o controle e prevenção desses fatores. Estudo documental, quantitativo, realizado com 165 prontuários de profissionais de enfermagem de hospital público de Fortaleza-CE. A análise se deu por estatística descritiva com dados expostos em quadros e tabelas. Nos resultados perceberam-se fatores de risco como antecedentes familiares com hipertensão arterial $(72,9 \%)$, sedentários $(64,9 \%)$, peso elevado $(56,4 \%)$, circunferência abdominal elevada $(49,7 \%)$, dentre outros. Para estratégias de promoção da saúde podem-se citar: orientações educativas, encaminhamentos e acompanhamento dos dados clínicos. Acredita-se na necessidade de atenção especial para tais profissionais, já que vivenciam uma profissão estressante, enfrentando problemas como insatisfação profissional, falta de reconhecimento financeiro e algumas doenças ocupacionais, o que pode prejudicar a qualidade de vida e dificultar o autocuidado.

Descritores: Doenças Cardiovasculares; Enfermagem; Promoção da Saúde.

ABSTRACT
The objectives of this study are to identify modifiable and non-modifiable risk factors to cardiovascular diseases present in professional nursing and to describe strategies for health promotion to control and prevent these factors. It is a documentary and quantitative study, conducted with 165 professional nursing records of a public hospital in Fortaleza-CE. The analysis was made using descriptive statistics, with data presented in charts and tables. As a result it was found risk factors such as family history of arterial hypertension $(72.9 \%)$, sedentary people $(64.9 \%)$, overweight $(56.4 \%)$, and high waist circumference $(49.7 \%)$, among others. Regarding health promotion strategies, it can be mentioned: educational guidance, referrals and follow-up clinical data. It is believed in the need for special attention to such professionals, since they experience a stressful profession, facing problems such as job dissatisfaction, lack of financial recognition and some occupational diseases, which may impair their quality of life and hinder self-care.

Key words: Cardiovascular Diseases; Nursing, Health Promotion.

\section{RESUMEN}

El estudio tuvo como objetivos: identificar los factores de riesgo, modificables y no modificables, para las enfermedades cardiovasculares presentes en los profesionales de enfermería y describir las estrategias de promoción de la salud para el control y la prevención de estos factores. Estudio documental, cuantitativo, realizado con 165 prontuarios de profesionales de enfermería de un hospital público de Fortaleza-CE. El análisis se dio con estadística descriptiva, con datos expuestos en cuadros y tablas. Como resultados se percibió que había factores de riesgo como antecedentes familiares con hipertensión arterial $(72,9 \%)$, sedentarios $(64,9 \%)$, peso elevado $(56,4 \%)$, circunferencia abdominal elevada $(49,7 \%)$, entre otros. Entre las estrategias de promoción de la salud se pueden citar: orientaciones educativas, encaminamientos y seguimiento de los datos clínicos. Se cree en la necesidad de atención especial para dichos profesionales, ya que viven una profesión estresante, enfrentando problemas como insatisfacción profesional, falta de reconocimiento financiero y algunas enfermedades ocupacionales, lo que puede perjudicar su calidad de vida y dificultar el auto-cuidado.

Palabras clave: Enfermedades Cardiovasculares; Enfermería; Promoción de la Salud.

\section{AUTOR CORRESPONDENTEＦernanda Jorge Magalhães E-mail: fernandajmagalhaes@yahoo.com.br}




\section{INTRODUÇÃO}

As doenças cardiovasculares (DCV) são alterações no funcionamento do sistema cardíaco, sendo este responsável por transportar oxigênio e nutrientes necessários às células para essas executarem suas tarefas ${ }^{(1)}$.

Tais doenças são consideradas um grande problema de saúde pública. Por serem a principal causa de morte em todo o mundo, em especial nas populações dos grandes centros urbanos $^{(2)}$. Dentre as DCV de maior ocorrência podem-se destacar doença arterial coronariana (DAC), insuficiência cardíaca, angina, infarto agudo do miocárdio (IAM), doenças valvares, arritmias, doenças hipertensivas, dentre outras.

Vários são os fatores de risco associados ao desenvolvimento de DCV, os quais podem ser modificáveis e não modificáveis. Os fatores de riscos modificáveis incluem hiperlipidemia, tabagismo, etilismo, hiperglicemia, obesidade, sedentarismo, má alimentação e uso de contraceptivos; e os não modificáveis incluem história familiar de DCV, idade, sexo e raça $a^{(3)}$.

No Brasil, as DCV figuram na lista das dez principais causas de morte, sendo que em 2007 houve uma taxa de 48,9\% de mortalidade específica por doenças do aparelho circulatório, e no Ceará esta taxa foi de $39,3 \%{ }^{(4)}$. Ressalta-se que, para a redução da incidência, prevalência e mortalidade destas doenças, faz-se necessário a prevenção dos fatores de risco associados a estes agravos.

Diante deste contexto, as DCV têm se destacado no âmbito da saúde pública, sendo propostos alguns programas de reabilitação cardiovascular e estratégias de promoção da saúde para a redução e/ou remoção de seus fatores de risco; assim como a possibilidade de minimizar a morbimortalidade, sobretudo da DAC e do IAM, além da boa relação entre custos sociais e econômicos destes programas, quando comparados ao tratamento medicamentoso e cirúrgico ${ }^{(2)}$.

Assim, uma empresa para alcançar a saúde ocupacional dos seus trabalhadores, precisa de um sistema de monitoramento dos programas da medicina preventiva ${ }^{(5)}$. As Normas Regulamentadoras (NR) da Segurança e Medicina do Trabalho viabilizam para a empresa medidas de prevenção de doença e promoção da saúde dos trabalhadores no local de trabalho, pois podem antecipar precocemente a situação de risco e danos à saúde do trabalhador ${ }^{(6)}$. Entre essas Normas, tem-se a NR-4 e a NR-7: a primeira estabelece a implantação dos Serviços Especializados em Segurança e Medicina do Trabalho (SESMT) e apresenta como finalidade a promoção da saúde e proteção a integridade do trabalhador no local de trabalho; a segunda estabelece a elaboração e implementação do Programa de Controle Médico e Saúde Ocupacional (PCMSO) e tem caráter preventivo, rastreamento e diagnóstico precoce dos agravos à saúde relacionados ao trabalho ${ }^{(6)}$.

O PCMSO é elaborado e executado pela equipe de saúde ocupacional, no qual são realizados os exames médicos ocupacionais, com base nos riscos à saúde. Além disso, é feito o monitoramento dos fatores de risco para as doenças crônico-degenerativas, como as DCV, e suas consequências na qualidade de vida do trabalhador. Também, são gerados relatórios clínicos e epidemiológicos, para que sejam planejadas e realizadas as ações de saúde, de acordo com a prevalência das doenças diagnosticadas. Deste modo, o PCMSO oferece subsídios que resultam em dados clínicos e epidemiológicos que fomentam as ações corretivas para melhorar a qualidade de vida dos trabalhadores no ambiente de trabalho.

Desta forma, prevenir, rastrear e diagnosticar fatores de risco das DCV nos profissionais, sobretudo nos da Enfermagem, exige acompanhamento rigoroso de equipe especializada, realização de estratégias educativas de modo a incentivá-los à adesão ao estilo de vida saudável, na perspectiva de diminuir e evitar as complicações dessas doenças. Especialmente nos hospitais de referência cardiopulmonar e acreditados pela Organização Nacional de Acreditação Hospitalar, espera-se possuir uma equipe de qualidade profissional e de maior experiência teórico-prática sobre tais condições de saúde.

Diante desse contexto, surgiram os seguintes questionamentos: quais os fatores de risco para DCV presentes em profissionais de enfermagem de um hospital público de Fortaleza com referência em cardiologia? Quais as estratégias de promoção da saúde desenvolvidas pelo SESMT para o controle e prevenção de DCV nos profissionais de enfermagem?

Assim sendo, este estudo teve como objetivos: identificar os fatores de risco modificáveis e não modificáveis para DCV presentes nos profissionais de enfermagem de um hospital público de referência e doenças cardiopulmonares e descrever as estratégias de promoção da saúde realizadas pelos profissionais do SESMT para o controle e a prevenção desses fatores de risco.

Espera-se com esta pesquisa contribuir para a sensibilização dos profissionais de enfermagem no controle e prevenção das DCV, minimizar as complicações a que estes estão expostos e colaborar para promoção da saúde dos profissionais que trabalham para promover a saúde da população.

\section{METODOLOGIA}

Estudo descritivo, documental, de natureza quantitativa, desenvolvido em um hospital público de nível terciário, referência no atendimento cardiovascular e pulmonar, situado em Fortaleza-Ceará-Brasil.

A população foi composta por 175 prontuários de profissionais de enfermagem da referida instituição, os quais foram convocados a realizar os exames para o Atestado Médico Ocupacional (ASO) no SESMT, realizado pela equipe de saúde ocupacional, composta por médico e enfermeira, no período de janeiro 2009 a dezembro de 2010. A amostra foi de 165 prontuários, obedecendo aos seguintes critérios de inclusão: ser profissional de enfermagem efetivo da instituição e estar exercendo suas atividades laborais no período de janeiro 2009 a dezembro de 2010. E como critérios de exclusão: prontuários com dados incompletos, funcionário com mais de um prontuário, tendo sido o mais antigo excluído.

A coleta de dados ocorreu em abril de 2011 nos prontuários da equipe de enfermagem arquivados no SESMT da instituição, nos quais há os dados estruturados de identificação do profissional, sexo, idade, estado civil, categoria e tempo de instituição 
sendo considerados como fatores de risco não modificáveis. Quanto às informações dos fatores de risco modificáveis investigou-se: sedentarismo, presença de diabetes mellitus (DM) e hipertensão arterial sistêmica (HAS), história familiar de HAS, tabagismo, etilismo, colesterol e triglicerídeos elevados, índice de massa corpórea (IMC), circunferência abdominal (CA) e pressão arterial (PA), conforme pressupostos das Diretrizes Brasileiras de Hipertensão. A coleta de informações sobre as estratégias de promoção da saúde foi realizada a partir dos documentos arquivados pela equipe de saúde ocupacional que atua no SESMT.

Os dados foram analisados por meio de estatística descritiva e da literatura pertinente à temática sendo apresentados em quadro e tabelas. A pesquisa foi aprovada pelo Comitê de Ética e Pesquisa da referida instituição, sob Parecer n 548/08.

\section{RESULTADOS}

Dentre os resultados encontrados, constatou-se um predomínio de profissionais jovens $(52,7 \%)$ com idades até 40 anos; a maioria do sexo feminino $(85,4 \%)$; quanto ao estado civil e número de filhos dos profissionais de enfermagem, percebeu-se que a maioria está solteira $(51,0 \%)$ e não tem nenhum filho $(43,0 \%)$ - Tabela 1 .

Tabela 1 - Fatores de risco cardiovascular não modificáveis nos profissionais de enfermagem. Fortaleza-CE, maio de 2011

\begin{tabular}{|c|c|c|c|}
\hline Características dos profissionais & $\mathbf{n}$ & $\%$ & Média \\
\hline \multicolumn{4}{|l|}{ Faixa etária } \\
\hline 24 a 29 anos & 24 & 14,5 & \\
\hline 30 a 40 anos & 63 & 38,2 & \\
\hline 41 a 50 anos & 52 & 32,0 & 40,4 anos \\
\hline 51 a 60 anos & 22 & 13,3 & \\
\hline$\geq 61$ anos & 4 & 2,42 & \\
\hline $\begin{array}{l}\text { Sexo } \\
\quad \text { Masculino }\end{array}$ & 24 & 14,5 & \\
\hline Feminino & 141 & 85,4 & \\
\hline \multicolumn{4}{|l|}{ Estado civil } \\
\hline Solteira(o) & 84 & 51,0 & \\
\hline Casada(o) & 68 & 41,2 & \\
\hline União consensual & 8 & 5,0 & \\
\hline Divorciada(o) & 5 & 3,1 & \\
\hline \multicolumn{4}{|l|}{ Número de filhos } \\
\hline Nenhum & 71 & 43,0 & \\
\hline Um & 30 & 18,2 & \\
\hline Dois & 44 & 26,6 & \\
\hline Três & 17 & 10,3 & \\
\hline Quatro & 3 & 1,81 & \\
\hline \multicolumn{4}{|l|}{ Função ocupacional } \\
\hline Enfermeira (o) & 15 & 9,1 & \\
\hline Técnica (o) de Enfermagem & 71 & 43,0 & \\
\hline Auxiliar de Enfermagem & 72 & 43,6 & \\
\hline Atendente de Enfermagem & 7 & 4,24 & \\
\hline \multicolumn{4}{|l|}{ Tempo de serviço na Instituição } \\
\hline 1 a 10 anos & 131 & 79,3 & \\
\hline 11 a 20 anos & 29 & 15,6 & 8,54 anos \\
\hline$\geq$ a 21 anos & 5 & 3,03 & \\
\hline
\end{tabular}

Fonte: Elaborado pelos próprios autores.
Ao analisar a função ocupacional destes profissionais percebe-se um predomínio de $43,6 \%$ de auxiliares de enfermagem e apenas $9,1 \%$ de enfermeiras(os); tendo sido identificado considerável número de profissionais de nível médio. Tais profissionais possuem, em média, 8,54 anos de tempo de serviço na Instituição.

Na Tabela 2 estão expostas as variáveis relacionadas aos fatores de riscos cardiovasculares modificáveis, cujos fatores mais presentes foram: sedentarismo $(64,9 \%)$, etilismo $(40,0 \%)$ e presença de HAS $(17,5 \%)$.

Tabela 2 - Caracterização dos profissionais de enfermagem quanto à presença de fatores de risco cardiovascular modificáveis. Fortaleza-CE, maio/2011

\begin{tabular}{lcc}
\hline Fatores de risco & $\mathbf{n}$ & $\mathbf{\%}$ \\
\hline Sedentarismo & 107 & 64,9 \\
Etilismo & 66 & 40,0 \\
HAS & 29 & 17,5 \\
Tabagismo & 18 & 10,9 \\
DM & 13 & 7,9 \\
\hline
\end{tabular}

Fonte: Elaborado pelos próprios autores.

Na Tabela 3, estão explicitadas as variáveis relacionadas ao IMC, CA, colesterol total, triglicerídeo e PA. Com relação ao IMC, $21,8 \%$ dos profissionais estavam obesos no dia da consulta, seguidos de 33,9\% com sobrepeso. A média do IMC foi de $27 \mathrm{Kg} / \mathrm{m}^{2}$. A CA de 49,7\% dos profissionais de enfermagem apresentou-se elevada.

O colesterol total elevado estava presente em 27,8\% dos profissionais de enfermagem, o triglicerídeo encontrava-se elevado em 25,5\%, e a pressão arterial de 8,5\% desses profissionais estava elevada no momento da aferição na consulta no SESMT.

Dentre as principais estratégias de promoção da saúde para os fatores de risco modificáveis, utilizada pela comissão do SESMT, na consulta ao profissional de enfermagem teve-se: abordagem educativa individual e em grupo com sensibilização para os efeitos nocivos do tabaco e etilismo incentivo à eliminação de seu uso; encaminhamento para grupo específico e/ou especialista como psicólogo, nutricionista e/ou cardiologista; sensibilização para a realização de atividades físicas e alimentação saudável, bem como relaxamento no ambiente laboral; acompanhamento periódico dos parâmetros clínicos e bioquímicos pelos profissionais do SESMT; ações educativas coletivas com entrega de material educativo como folders e verificação da pressão arterial, mensuração do IMC, colesterol e triglicerídeos.

Verificou-se que dentre as estratégias educativas para as DCV, a maioria sugere abordagens individuais e grupais. As abordagens individuais são realizadas no momento do ASO e, dependendo das atividades laborais dos profissionais, são feitas em periodicidade semestral ou anual, direcionadas para a prática do autocuidado. As ações coletivas são desempenhadas de acordo com as datas comemorativas da área da saúde, ou conforme a presença dos altos índices dos fatores cardiovasculares. 
Tabela 3 - Caracterização dos profissionais de enfermagem quanto aos fatores de risco cardiovascular modificáveis. Fortaleza-CE, maio/2011

\begin{tabular}{|c|c|c|c|}
\hline Fatores de risco & $\mathbf{N}$ & $\%$ & Média \\
\hline \multicolumn{4}{|l|}{ IMC } \\
\hline Normal $\left(\geq 20 \mathrm{~kg} / \mathrm{m}^{2}\right)$ & 15 & 9,1 & \\
\hline Sobrepeso ( $>$ a 25 e $<30 \mathrm{~kg} / \mathrm{m}^{2}$ ) & 58 & 35,1 & \\
\hline Obesidade $\left(\geq\right.$ a 30 e $\left.<35 \mathrm{~kg} / \mathrm{m}^{2}\right)$ & 56 & 33,9 & $27 \mathrm{~kg} / \mathrm{m}^{2}$ \\
\hline Obesidade mórbida ( $\geq 35 \mathrm{~kg} / \mathrm{m}^{2}$ ) & 36 & 21,8 & \\
\hline \multicolumn{4}{|l|}{ CA } \\
\hline Normal ( $<88 \mathrm{~cm}$ para mulheres e $<102 \mathrm{~cm}$ para homens) & 83 & 50,3 & \\
\hline Elevado ( $>88 \mathrm{~cm}$ para mulheres e $>102 \mathrm{~cm}$ para homens) & 82 & 49,7 & $92 \mathrm{~cm}$ \\
\hline \multicolumn{4}{|l|}{ Colesterol Total } \\
\hline Normal $(\leq 200 \mathrm{mg} / \mathrm{dl})$ & 119 & 72,2 & \\
\hline Elevado (> 200mg/dl) & 46 & 27,8 & \\
\hline \multicolumn{4}{|l|}{ Triglicerídeo } \\
\hline Normal $(\leq 150 \mathrm{mg} / \mathrm{dl})$ & 123 & 74,5 & \\
\hline Elevado ( $\geq 150 \mathrm{mg} / \mathrm{dl})$ & 42 & 25,5 & \\
\hline \multicolumn{4}{|l|}{ Pressão Arterial Sistêmica (PAS) } \\
\hline Ótima ( $\leq 120 \times 80 \mathrm{mmHg}$ ) & 109 & 66,0 & \\
\hline Normal ( $\leq 130 \times 85 \mathrm{mmHg})$ & 24 & 14,5 & \\
\hline Normal limítrofe $(130-139 \times 85-89 \mathrm{mmHg})$ & 18 & 11,0 & \\
\hline Hipertensão estágio 1 (140-159 x 90-99 mmHg) & 10 & 6,1 & \\
\hline Hipertensão estágio 2 (160-179 x 100-109mmHg) & 3 & 1,8 & \\
\hline Hipertensão estágio 3 ( $\geq 180 \times 110 \mathrm{mmHg}$ ) & 1 & 0,6 & \\
\hline
\end{tabular}

Fonte: Elaborado pelos próprios autores.

Em todas essas abordagens são desenvolvidas estratégias de sensibilização para adesão de estilo de vida saudável, incluindo atividades físicas e alimentação saudável, bem como mudança de comportamento e, principalmente, praticar o autocuidado para uma melhor qualidade de vida, além de encaminhamentos para especialistas como cardiologista, nutricionista e, muitas vezes, psicólogo.

\section{DISCUSSÃO}

Descrever os indicadores de caracterização dos participantes da amostra é fundamental para identificar os profissionais de Enfermagem que foram atendidos pelo SESMT, além de saber a concordância dos dados com a literatura. Como indicadores de caracterização dos profissionais de enfermagem foram considerados os fatores de risco modificáveis e não modificáveis.

Para os fatores de risco modificáveis teve-se: faixa etária, sexo, estado civil, número de filhos, função ocupacional e tempo de serviço na instituição que pode ser relacionado com o estresse e possíveis alterações na qualidade de vida. A análise dos dados dos profissionais de enfermagem foi realizada simultaneamente, já que a literatura traz os mesmos dados de forma associada.

Com relação à faixa etária dos profissionais constatou-se um predomínio de idade entre 30 e 40 anos, tal fator não deve ser encarado como meramente biológico ou não influenciador, pois o avançar da idade pode contribuir para o aparecimento de fatores de risco não modificáveis e complicações a curto e longo prazo para as DCV.
Considera-se a idade biológica e cronológica, o perfil de longevidade familiar, a associação com outros fatores de risco comuns nessa faixa etária (hipertensão arterial e diabetes), a presença de doença arterial, o estado físico geral, as doenças crônicas associadas e as interações medicamentosas. Deve-se lembrar, ainda, a importância do exercício físico, como medida associada de promoção da saúde ao controle dos fatores de risco, respeitando as limitações próprias da idade, uma vez que qualquer redução do risco tem importante repercussão na morbimortalidade ${ }^{(7)}$.

Corrobora-se com estudiosos ${ }^{(8)}$ acerca da gravidade das DCV e do aumento de sua incidência com o avanço da idade, pois grande parte dessas doenças poderia ser evitada. Para as doenças crônicas não transmissíveis mais frequentes como diabetes e câncer as quais compartilham vários fatores de risco com as DCV, propõe-se uma abordagem de prevenção e controle integrados, em todas as idades, baseada na redução dos seguintes fatores como: hipertensão arterial, tabagismo, uso de bebidas alcoólicas, falta de atividade física, dieta inadequada, obesidade e hipercolesterolemia.

Com relação ao sexo verificou-se um predomínio de muIheres, podendo considerar a Enfermagem, historicamente, como uma profissão feminina, porque é exercida majoritariamente por mulheres e porque o cuidado, objeto prioritário dessa profissão, tem sido tradicionalmente de responsabilidade social também das mulheres, assim como favorecem o aumento da inserção destas no mercado de trabalho ${ }^{(9)}$.

No entanto, o presente estudo limita-se ao predomínio de sexo feminino, talvez devido sua prevalência na profissão de enfermagem. Porém, com relação aos fatores de risco cardiovasculares, verifica-se um predomínio de estudos que referem o sexo masculino como um achado esperado, tendo em vista que os homens apresentam alterações cardíacas mais precocemente que as mulheres, o que favorece o surgimento de complicações, como doença arterial coronariana ${ }^{(7)}$.

Quanto à função ocupacional predominou profissionais de nível médio com mais de oito anos de tempo de serviço, o que se remete a extensa experiência com a apresentação dos fatores de risco para as doenças cardiovasculares. Tais achados podem ser decorrentes do maior quantitativo desse profissional na equipe de enfermagem.

No entanto, remete-se a uma reflexão já que em outro estudo $^{(10)}$, realizado na mesma instituição, constatou-se que tais profissionais tiveram predomínio de fatores de risco para o desenvolvimento de HAS, já que seria esperado encontrar menor prevalência da doença, em virtude da equipe de enfermagem 
ter oportunidade de adquirir informações acerca da patologia, tratamento e prevenção, e ainda tem o papel de disseminá-las. Especialmente, por se tratar de profissionais que trabalham em um hospital e referencia nas áreas cardiovascular, torácica e pulmonar, e atuar como centro de ensino e pesquisa.

\section{Fatores de risco cardiovasculares modificáveis}

Com relação ao tabagismo, $11 \%$ dos trabalhadores de enfermagem eram tabagistas. Sabe-se que o fumo é considerado o único fator de risco totalmente evitável para o surgimento de doenças e morte cardiovasculares ${ }^{(7-11)}$.

De acordo com a ingestão de bebidas alcoólicas, verificou-se que, no presente estudo, $40 \%$ dos profissionais de enfermagem faziam uso com freqüência e ultrapassavam a quantidade de $30 \mathrm{~g}$ de etanol ao dia, para homens, e metade desta quantidade para mulheres. Estudos comprovam que o etilismo é considerado importante fator de risco para elevação da PA; sendo referido que o consumo de bebida alcoólica pode aumentar consideravelmente o risco de doenças cardiovasculares ${ }^{(7)}$.

No sistema cardiovascular, o consumo elevado e frequente de álcool está associado ao aumento da pressão arterial, desrregulação de lipídios e triglicerídeos e maior risco de infarto do miocárdio e doenças cerebrovasculares. O álcool também eleva a frequência cardíaca de consumidores eventuais, aumentando o desgaste cardíaco em repouso e o consumo energético pelo miocárdio ${ }^{(12)}$.

Com relação ao sedentarismo, definido como a ausência de esforço físico no trabalho ou no lazer ${ }^{(12)}$, a amostra estudada relatou a não realização de atividade física. Portanto, corrobora-se com estudiosos, que referem à importância da prática de exercícios físicos com finalidade de evitar o surgimento de DCV, pois além de melhorar a qualidade de vida do paciente, aumenta as taxas do colesterol HDL e diminui o sobrepeso ${ }^{(11-13)}$.

A morbidade por DM estava presente em 7,9\% dos trabalhadores, sendo associado à HAS com 17,5\% dos profissionais de enfermagem. Um estudo desenvolvido no Rio Grande do Sul investigou 93 portadores de HAS constatando que $23,9 \%$ dos entrevistados eram portadores de DM. Além disso, relatos de estudiosos enfatizam que a presença de morbidades associadas à HAS, principalmente, o DM aumenta os riscos de um acidente vascular encefálico ${ }^{(14)}$.

O fator não modificável, como a presença de HAS, foi bastante prevalente na pesquisa, com $17,5 \%$ dos profissionais que possuem historia familiar de HAS, o que está de acordo com um estudo realizado em 2008, em que se identificou que $32 \%$ dos pacientes entrevistados tinham história familiar de $\mathrm{HAS}^{(15)}$.

No que diz respeito aos exames físicos e bioquímicos, verifica-se que o colesterol total está alto em $27,8 \%$ dos profissionais no presente estudo. Já o triglicerídeo estava alterado em 25,5\%. As dislipidemias são consideradas como um problema de saúde pública devido a sua estreita relação com o surgimento de DCV, estando classificadas entre os mais importantes fatores de risco para doença cardiovascular aterosclerótica ${ }^{(16)}$.

A PA dos trabalhadores estava elevada no momento da consulta ao SESMET. Com relação ao IMC a maioria estava com sobrepeso, seguidos de obesos no dia da consulta de Enfermagem. Por isso, corrobora-se com autores ${ }^{(17)}$ que referem que as alterações do IMC podem estar diretamente relacionadas com a ocorrência de HAS.

Dados provenientes de 48 diferentes países entre 1983 e 1986 mostraram que entre $50 \%$ e $70 \%$ da população de adultos com idades entre 35 e 64 anos estavam acima do peso ou eram obesos $^{(18)}$. No que diz respeito a CA, 49,7\% dos trabaIhadores estavam com a CA acima da média permitida. A média da CA foi de $92 \mathrm{~cm}$. O depósito de gordura no abdômen contribui com o aumento do risco de doenças cardiovasculares. A associação do IMC e da circunferência abdominais ambos alterados aumenta as chances de ocorrência de $\mathrm{HAS}^{(17)}$.

Diante dos resultados da presente pesquisa, percebe-se que esses assinalam a importância da identificação e quantificação dos diferentes fatores de risco para DCV em desenvolver ações relevantes na intervenção preventiva para essa doença. Percebe-se que há semelhanças na prevalência da doença em profissionais de enfermagem. Tal fato demonstra que a auto-responsabilidade e o autocuidado constituem habilidades essenciais para a superação de doenças em qualquer profissão(19).

\section{Estratégias de promoção da saúde para minimizar os fa- tores de DCV}

Atualmente, as transformações no ambiente da atenção à saúde exigem o uso de estratégias de educação em saúde, de forma que os indivíduos tenham a capacidade de atender as suas necessidades. Uma educação para a saúde eficaz volta-se para o bem estar das pessoas e das comunidades e transcorre por processos de ensino e aprendizagem.

O ensino com ênfase na educação para saúde tem origem, em parte, do direito das pessoas terem acesso a uma atenção a saúde compreensiva, possibilitando o surgimento de um público informado que questiona mais significativamente sobre saúde e sobre os serviços de atenção a saúde que recebem. Um dos maiores grupos de pessoas que requerem educação para a saúde é aqueles com doenças crônicas e, à medida que aumenta a longevidade da população, aumenta também as pessoas portadoras dessas doenças. E, com o avanço das novas terapêuticas, as pessoas necessitam adotar comportamentos mais conscientes ${ }^{(3)}$.

No processo do cuidar, a equipe de enfermagem atua como cuidador do outro e, na maioria das vezes, se esquece de praticar o autocuidado. Vários fatores concorrem para essa problemática, dentre estes se tem a dupla jornada de trabaIho, a responsabilidade muitas vezes de garantir a manutenção da família, e outros compromissos referentes às condições econômicas. Todas contribuem para o surgimento de DCV, incluindo ainda o estresse advindo do ambiente de trabalho e o constante desgaste emocional em face desse convívio ${ }^{(10)}$.

Diante dessa realidade, a equipe de saúde ocupacional, no momento do ASO, identifica os principais fatores de risco cardiovasculares e propõe algumas estratégias de promoção, proteção e recuperação da saúde junto aos profissionais de enfermagem, tais como acompanhamento individual e em grupo, assim como ações de sensibilização para modificar hábitos considerados não saudáveis como uso de tabaco e bebidas alcóolicas, alimentação hipercalórica e a não adesão da prática de atividade física. 
Corroboram-se com os autores ${ }^{(20)}$ quando referem à importância de uma prática profissional, em especial dos que atuam no SESMT, viabilizando a realização de atividades educativas com base na comunicação, favorecendo a sensibilização e a emancipação do trabalhador, uma vez que a educação em saúde possibilita a criação do vínculo profissional-paciente, reafirmando condições para modificações comportamentais e a redução ou extinção dos fatores de risco cardiovasculares em profissionais de enfermagem atuantes em um hospital referência em doenças cardiopulmonares.

\section{CONCLUSÃO}

As características sociodemográficas dos profissionais de enfermagem verificou-se que a maioria era do sexo feminino, com média de idade 40,4 anos, solteira, sem filhos, exerce atividade ocupacional de técnicas de enfermagem e com tempo de atuação na instituição entre um a dez anos e 72,9\% com antecedentes familiares de HAS sendo considerados fatores de risco não modificáveis.

No que se refere à presença de fatores de risco cardiovasculares modificáveis constatou-se o sedentarismo $(64,9 \%)$, peso elevado (56,4\%), 49,7\% com CA acima dos níveis de normalidade; ingestão de algum tipo de bebida alcoólica com frequência $(40 \%)$, o colesterol total elevado $(27,8 \%)$, triglicerídeo elevado (25,5\%), diagnóstico de HAS $(17,5 \%)$, tabagistas $(11,0 \%)$, PA elevada $(8,5 \%)$, e diagnóstico de DM $(7,9 \%)$.

Em relação às estratégias educativas para prevenção e controle dos fatores de riscos das DCV, percebe-se que a equipe de saúde ocupacional realiza de forma individual e coletiva, além de encaminhamentos para os especialistas, especialmente os que apresentam maior risco, estes são acompanhados e sensibilizados para aderir um estilo de vida saudável e praticar mudança de comportamento para uma melhor qualidade de vida.

O presente estudo reflete a importância de pesquisas acerca de fatores de riscos para DCV em profissionais de enfermagem, gerando informações importantes para os gestores das instituições de saúde, além de favorecer a elaboração de estratégias de promoção da saúde, que atendam às características específicas desse grupo, promovendo assim a saúde do cuidador.

Apesar do empenho da equipe de saúde ocupacional, estes achados justificam a necessidade de maior comprometimento das autoridades locais, assim como da própria equipe de saúde ocupacional do SESMT da instituição, pois se percebe um alto índice de fatores de risco para DCV presentes nos profissionais da enfermagem.

Vale salientar, a importância de intensificar a implementação de ações interventivas integradas e intersetoriais de promoção da saúde e prevenção de DCV, de forma individual e coletiva, auxiliando as pessoas a modificarem os comportamentos de risco, favorecendo, assim, a adoção de hábitos de vida mais saudáveis.

Ao focalizar na Enfermagem, considerada uma profissão que se compromete com a saúde do ser humano e sua qualidade de vida, percebe-se que estes necessitam de maior autoestima e preocupação com a própria saúde; ressaltando que os profissionais de enfermagem são vulneráveis aos fatores que predispõem às DCV.

Dentre as limitações do estudo, podem ser citados os registros incompletos da consulta de enfermagem sobre os dados clínicos e sociodemográficos dos profissionais, podendo este ser um aspecto contribuinte para mascarar os reais fatores de risco para as doenças cardiovasculares.

Ressalta-se a importância de estratégias que promovam a saúde e favoreçam a qualidade de vida desses profissionais, pois em geral são cuidadores, entretanto, têm dificuldades de praticar o autocuidado. Faz necessário acompanhamento e atenção especial para todos os profissionais da saúde, de maneira especial os de enfermagem, uma vez que o processo de trabalho desenvolvido pelos mesmos envolve riscos ocupacionais, longas jornadas e excesso de estresse. Torna-se, portanto, prerrogativa das políticas de saúde priorizar a atenção aos profissionais cuidadores no âmbito das Instituições de saúde, com vistas a reduzir os riscos cardiovasculares e promover a saúde.

\section{REFERÊNCIAS}

1. Ribeiro PRQ, Oliveira DM. Reabilitação cardiovascular, doença arterial coronariana e infarto agudo do miocárdio: efeitos do exercício físico. Rev Digital [Internet]. 2011. [acesso em 12 Jun 2012];15(152):1. Disponível em: http://www.efdeportes.com/efd152/reabilitacao-cardiovascular-efeitos-do-exercicio-fisico.htm

2. Iglesias CMF, Jesus JA, Santiago LC, Santoro LC. A importância da sistematização da assistência de enfermagem no cuidado ao cliente portador de infarto agudo do miocárdio. Rev Pesqui Cuid Fundam. 2010 Out-Dez;2(Suppl.):974-7.

3. Smeltzer SC, Bare BG. Histórico da função cardiovascular. In: Smeltzer SC, Bare BG. Brunner e Suddarth: Tratado de enfermagem médico-cirúrgica. 10. ed. Rio de Janeiro: Guanabara Koogan; 2009. p. 682-700.
4. Ministério da Saúde. Indicadores de mortalidade. C.8 Taxa de mortalidade específica por doenças do aparelho circulatório [Internet]. Brasília: Ministério da Saúde; 2011 [acesso em 12 Abr 2012]. Disponível em: http://tabnet.datasus.gov.br/cgi/ tabcgi.exe?idb2009/c08.def

5. Bulhões I. Enfermagem do Trabalho. Rio de Janeiro: Ideas; 1996.

6. Ministério da Saúde. Segurança e Medicina do Trabalho. 2. ed. São Paulo: Saraiva; 2008.

7. Lima V, Caetano JA. Soares E, Santos ZMSA. Fatores de risco associados à hipertensão arterial sistêmica em vitimas de acidente vascular cerebral. Rev Bras Promoç Saúde. 2006;19(3):149-54. 
8. Pereira JC, Barreto SM, Passos VMA. Perfil de risco cardiovascular e autoavaliação da saúde no Brasil: estudo de base populacional. Rev Panam Salud Pública. 2009 Jun;25(6):491-8.

9. Aperibense PGGS, Barreira IA. Nexos entre Enfermagem, Nutrição e Serviço Social, profissões femininas pioneiras na área da Saúde. Rev Esc Enferm USP. 2008 Set;42(3):474-82.

10. Custódio IL, Lima FET, Almeida MI, Silva LF, Monteiro AR. Perfil sociodemográfico e clínico de uma equipe de enfermagem portadora de Hipertensão Arterial. Rev Bras Enferm. 2011 Jan-Fev;64(1):18-24.

11. Sociedade Brasileira Hipertensão. VI Diretrizes Brasileiras de Hipertensão Arterial. São Paulo: SBC; 2010.

12. Carnelosso ML, Barbosa MA, Porto CC, Silva SA, Carvalho MM, Oliveira ALI. Prevalência de fatores de risco para doenças cardiovasculares na região leste de Goiânia (GO). Ciênc Saúde Coletiva. 2010;15(Suppl.1):1073-80.

13. Ferreira Filho C, Meneghini A, Riera ARP, Serpa Neto AS, Teixeira GK, Ferreira C. Benefícios do exercício físico na hipertensão arterial sistêmica. Arq Méd ABC. 2007 Jul-Dez;32(2):82-7.

14. Renner SBA, Franco RR, Berlezi EM, Bertholo L. Associação da hipertensão arterial com fatores de riscos cardiovasculares em hipertensos de ljuí, RS. Rev Bras Anal Clin. 2008;40(4):261-6.
15. Araújo APS, Silva PCF, Moreira RCPS, Bonilha SF. Prevalência dos fatores de risco em pacientes com acidente vascular encefálico atendidos no setor de neurologia da clínica de fisioterapia da UNIPAR, campus sede. Arq Ciências Saúde UNIPAR. 2008 Jan-Abr;12(1):35-42.

16. Fagherazi, S, Dias RL, Bortolon F. Impacto do exercício físico isolado e combinado com dieta sobre os níveis Séricos de HDL, LDL, colesterol total e triglicérides. Rev Bras Med Esporte. 2008 Jul-Ago;14(4):381-6.

17. Sarno F, Monteiro CA. Importância relativa do índice de massa corporal e da circunferência abdominal na predição da hipertensão arterial. Rev Saúde Pública. 2007 Out;41(5):788-96.

18. Eyken EBBDV, Moraes CL. Prevalência de fatores de risco para doenças cardiovasculares entre homens de uma população urbana do Sudeste do Brasil. Cad Saúde Pública. 2009 Jan;25(1):111-23.

19. Maia CO, Goldemeier S, Moraes MA, Boaz MR, Azzolin K. Fatores de risco modificáveis para doença arterial coronariana nos trabalhadores de enfermagem. Acta Paul Enferm. 2007 Abr-Jun;20(2):138-42.

20. Costa JA, Balga RSM, Alfenas RCG, Cotta RMM. Promoção da saúde e diabetes: discutindo a adesão e a motivação de indivíduos diabéticos participantes de programas de saúde. Ciênc Saúde Coletiva. 2011; 16(3):2001-9. 